\title{
Recurrent intradural spinal tuberculosis abscess in a child - follow-up magnetic resonance imaging
}

Mustafa Karabina, Özlem Korkmaz, Sinem Aydın, Bahar Atasoy, Alpay Alkan

Department of Radiology, School of Medicine, Bezmialem Vakif University, Istanbul, Turkey

Submitted: 30 June 2016

Accepted: 24 August 2016

Arch Med Sci Civil Dis 2016; 1: e75-e78

DOI: 10.5114/amscd.2016.62136

Copyright $\odot 2016$ Termedia \& Banach

Tuberculosis (TB) is a common cause of spinal infection in underdeveloped and developing countries. Immune-compromised patients are also affected by spinal TB infection in developed countries. A small number of cases with abscess of the spinal cord due to tuberculosis have been reported in the literature [1-3]. It may cause neurological symptoms as seen in spinal cord neoplasms. Because of the good response to quick treatment, early diagnosis is very important for sufficient recovery of symptoms. We report a case of a child with a recurrent spinal intradural tuberculosis abscess, which is seen rarely in childhood.

The 7-year-old female patient had a history of surgery due to spinal tuberculosis abscess. Gram stains for bacteria were negative, but acid-alcohol-fast bacilli were found in the specimen. A diagnosis of tuberculosis was made based on the microbiological studies. Tuberculosis antibiotherapy (isoniazid + rifampicin + pyrazinamide + ethambutol) was started after the surgery, but treatment had to stopped due to increasing liver enzymes (ALT, AST, LDH, GGT) after 2 months. Three months after the surgery she was referred to our hospital pediatric clinic with fever, hip pain and abdominal pain and also urinary incontinence. There was no spinal tenderness, no headache, no stiff neck, and no weakness of lower extremities. Knee and ankle jerks were normoactive bilaterally. Sensory examination was normal. Laboratory examination showed a white blood cell count (WBC) of $8.55 / \mathrm{mm}^{3}$, hemoglobin $12 \mathrm{~g} / \mathrm{dl}$ and an erythrocyte sedimentation rate (ESR) of $11 \mathrm{~mm} / \mathrm{h}$, C-reactive protein (CRP) $1,2 \mathrm{mg} /$ dl. Rheumatologic tests were normal. Grade 1 pelvicalyceal ectasia was seen on ultrasound and grade 4-5 vesicoureteral reflux (VUR) was detected on the voiding cystourethrogram. The contrast-enhanced magnetic resonance imaging (MRI) study of the lumbar spine revealed expansion of the spinal cord due to peripherally enhancing lesions which extended for a distance of approximately $6.8 \mathrm{~cm}$ between $\mathrm{L} 2$ and $\mathrm{S} 1$. In the intradural space between the levels of L2 and S1 there was a heterogeneous T2 hyperintense lesion which expanded the spinal cord. The lesion was predominantly hypointense on the T1W sequence and demonstrated thick irregular peripheral enhancement on contrast-enhanced T1 images. Cauda equina fibers and leptomeninges also enhanced after contrast administration (Figures 1 A, B). Intradural tuberculosis abscess was diagnosed based on clinical and MRI findings. The abscess was drained surgically and a new antituberculosis therapy (rifampicin + clarithromycin + ethambutol) was started by pediatricians because of hepatotoxicity of previously given anti-TB drugs. After the therapy, follow-up imaging stud-

\author{
Corresponding author: \\ Mustafa Karabina MD \\ Department of Radiology \\ School of Medicine \\ Bezmialem Vakif University \\ Vatan Street, Fatih \\ 4600 Istanbul, Turkey \\ Phone: +9005544320240 \\ Fax: +9005544320240 \\ E-mail: mustafakarabina30@ \\ gmail.com
}



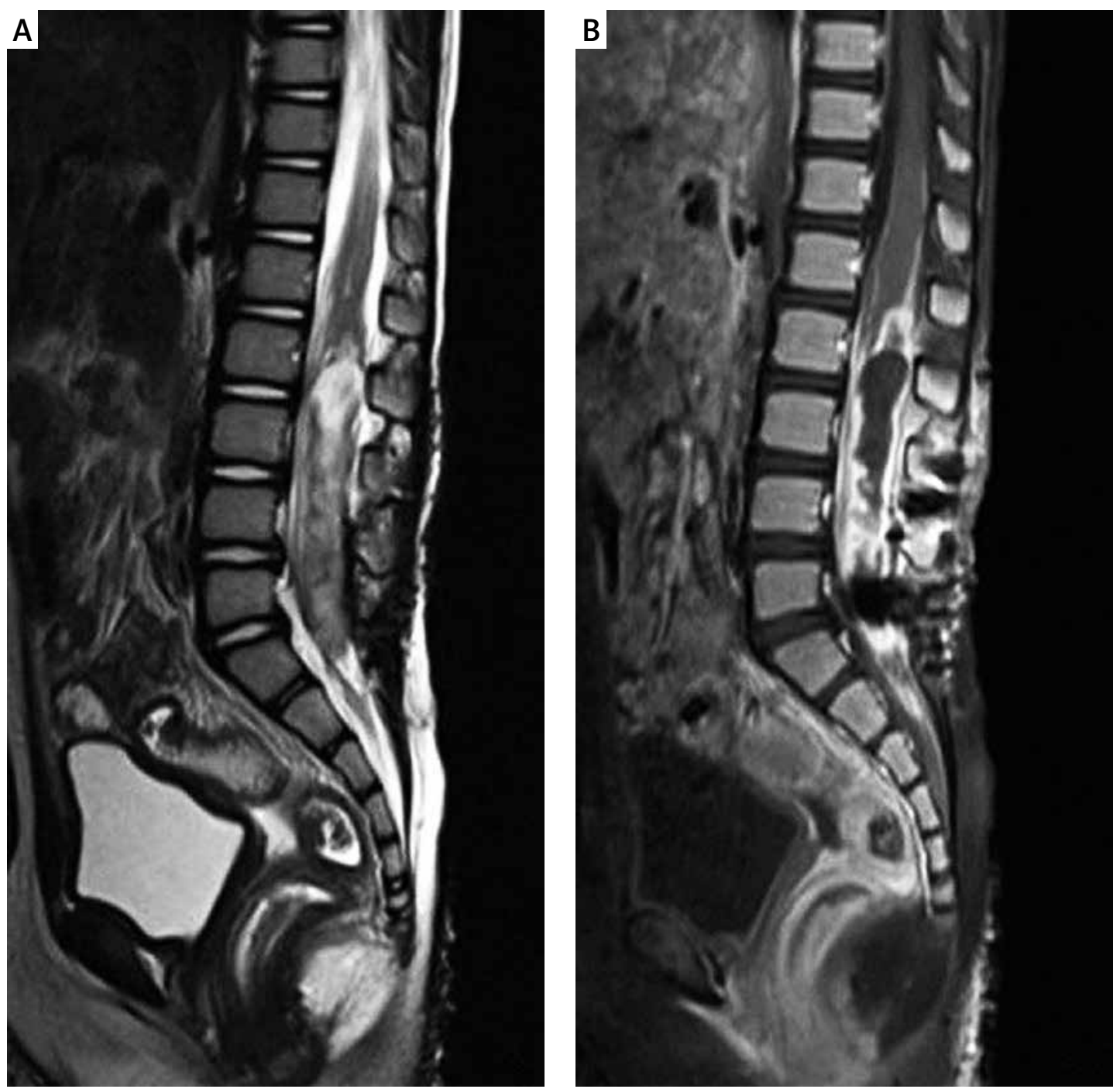

Figure 1. A - Sagittal T2 image shows that in the intradural space between the levels of $L 2$ and $S 1$ there was a heterogeneous T2 hyperintense lesion which expanded the spinal cord. B - Sagittal T1 C+ image shows expansion of the spinal cord due to peripherally enhancing lesions which extended between L2 and S1. Cauda equina fibrilles and leptomeninges also enhanced after contrast administration

ies showed prominent regression of the contrast enhancement and size of the lesion, as well as the degree of the cord expansion. Leptomeningeal enhancement was also decreased (Figures 2 A, B). Three months later she was referred to our emergency service again with leg and hip pain. The contrast-enhanced MRI study of the lumbar spine showed a recurrent abscess at the L1-S1 level. The lesion revealed cystic, necrotic and hemorrhagic areas. After contrast administration enhancing leptomeninges extended superiorly until the T8 level (Figures $3 \mathrm{~A}, \mathrm{~B}$ ).

Tuberculosis is a chronic granulomatous infection caused by Mycobacterium tuberculosis. The central nervous system (CNS) is affected in about $10 \%$ of all TB cases and it frequently presents as meningitis, tuberculoma or abscess formation [4]. Dissemination of TB into the CNS occur via 3 different ways. The most common type is he- matogenous spread from pulmonary TB. The other 2 ways are caudal extension from cranial TB and local extension from vertebral body (Pott's disease), facet joint or intervertebral disc TB infection.

Spinal cord involvement due to TB is usually characterized by spondylodiscitis, especially at the lower thoracic and higher lumbar level, and granuloma formation in the spinal cord. Spinal cord TB abscess is a rare clinical entity; few cases have been reported in the literature [1-3]. The clinical presentation of spinal cord TB abscess is characterized by fever and back pain, and, according to the localization of the abscess in the spinal cord, shoulder, hip or extremity pain can occur. If spinal cord abscesses are in a chronic state, symptoms can mimic spinal cord tumors. Leukocytosis, increased ESR and CRP are laboratory findings of TB infection. Positive acid-fast ba- 

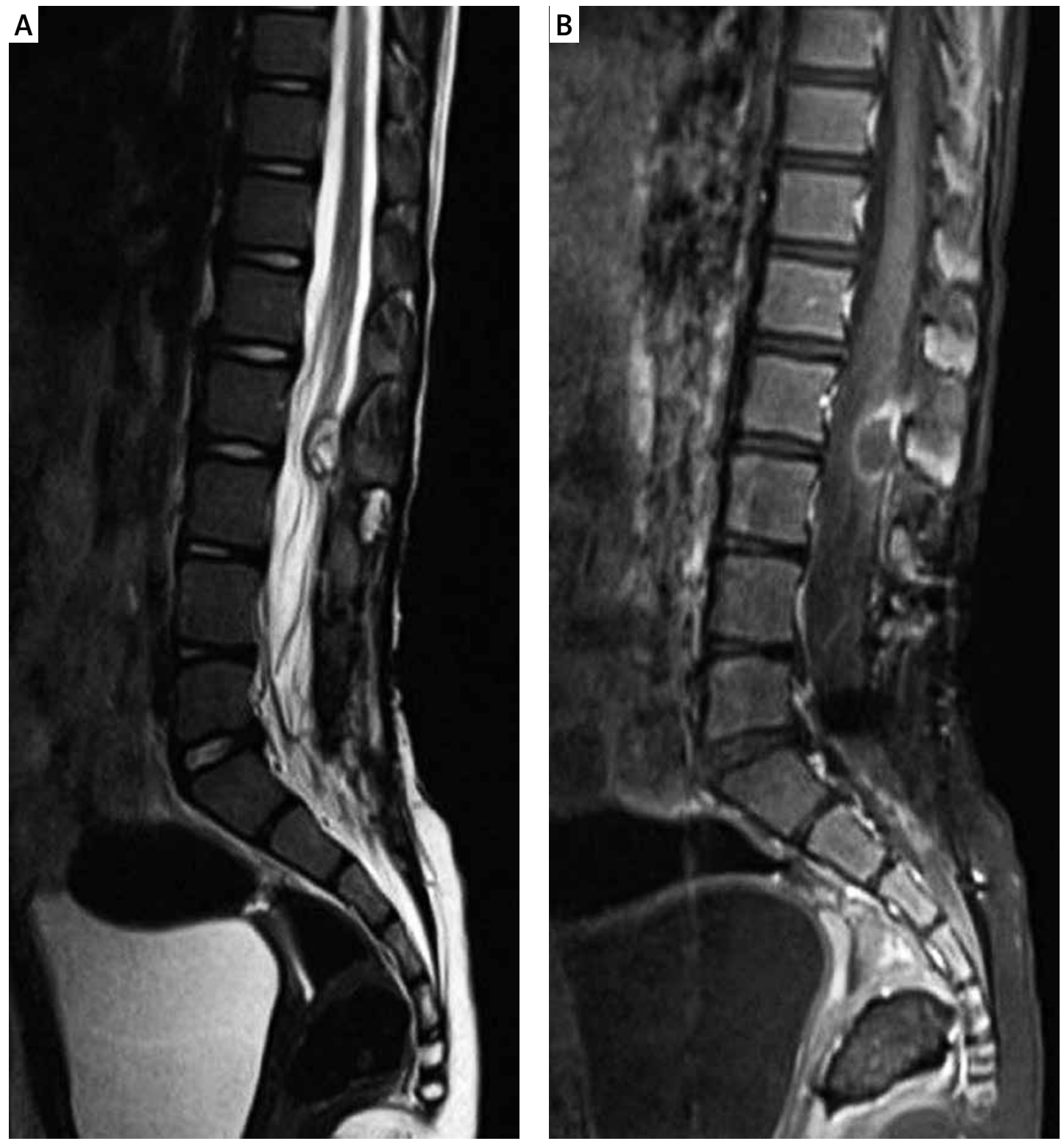

Figure 2 A, B. Sagittal T2 images and sagittal T1 images after contrast administration show prominent regression of the contrast enhancement and the size of the lesion, as well as the degree of cord expansion. Leptomeningeal enhancement was also decreased

cilli or culture examinations reveal the causative agent of the abscess.

Various imaging modalities can be used to detect spinal TB such as scintigraphy, computed tomography (CT), and myelography, but the most useful and sensitive method is MRI for both diagnosis and follow-up imaging. Abscess localization, extension and association with adjacent anatomical structures can be seen by MRI. The TB abscess demonstrates a hypointense signal on T1W sequences and a hyperintense signal on $\mathrm{T} 2 \mathrm{~W}$ sequences. Spinal cord expansion and capsular ring formation in contrast-enhanced series are typical MRI features of the TB abscess [5]. These findings are very useful to differentiate an abscess from syrinx or myelomalacia. However, a TB abscess cannot easily be differentiated from intramedullary tuberculoma.

For treatment of an intramedullary TB abscess, initially medical antibiotherapy should be given without surgical intervention. However, if neurological symptoms progresses rapidly, surgery should be considered. The most appropriate treatment approach to a TB abscess is both surgical drainage and tbc antibiotherapy. A spinal tuberculosis abscess can recur despite the surgical and medical therapy. Especially incomplete surgical drainage or interruption of antibiotherapy increases the abscess recurrence rate. In our case the TB abscess recurred one month after interruption of antibiotherapy due to liver toxicity. 

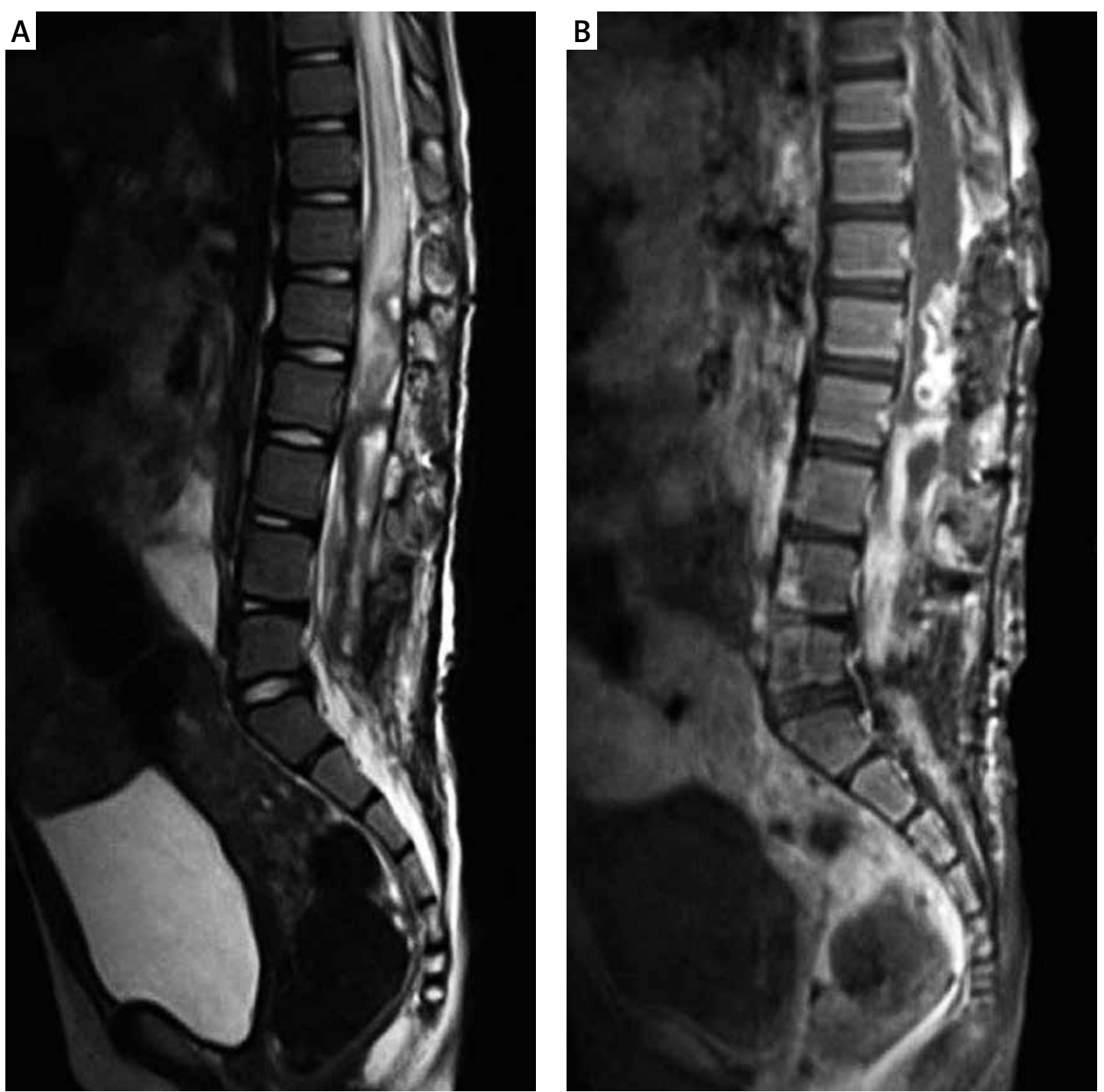

Figure 3. A - Sagittal T2 image shows recurrent abscess at L1-S1 level. Lesion revealed cystic, necrotic and hemorrhagic areas. B - Sagittal T1 image after contrast administration shows enhancing leptomeninges extending superiorly until T8 level

Magnetic resonance imaging should be the first choice of imaging modality in both the diagnosis and the follow-up of spinal tuberculosis. Clinicians and radiologists should always consider the intradural spinal abscess form of TB to avoid irreversible sequelae in the presence of known previous history of TB.

\section{Conflict of interest}

The authors declare no conflict of interest.

\section{References}

1. Desai KI, Muzumdar DP, Goel A. Holocord intramedullary abscess: an unusual case with review of literature. Spinal Cord 1999; 37: 866-70.

2. Hanci M, Sarioglu AC, Uzan M, Islak C, Kaynar MY, Oz B. Intramedullary tuberculous abscess: a case report. Spine (Phila Pa 1976) 1996; 21: 766-9.
3. Woolsey RM, Chambers TJ, Chung HD, McGarry JD. Mycobacterial meningomyelitis associated with human immunodeficiency virus infection. Arch Neurol 1988; 45: 691-3.

4. Murthy JM. Tuberculous meningitis: the challenges. Neurol India 2010; 58: 716-22.

5. Khalid M, Khalid S, Mittal S, Ahmad U. Intramedullary tubercular abscess with syrinx formation. J Pediatr Neurosci 2012; 7: 61-3. 